J. Korean Math. Soc. 42 (2005), No. 4, pp. 709-721

\title{
NOTES ON SELECTION PRINCIPLES IN TOPOLOGY (I): PARACOMPACTNESS
}

\author{
L. Babinkostova, LJ. D. R. Kočinac, And M. Scheepers
}

\begin{abstract}
G. Gruenhage gave a characterization of paracompactness of locally compact spaces in terms of game theory ([6]). Starting from that result we give another such characterization using a selective version of that game, and study a selection principle in the class of locally compact spaces and its relationships with game theory and a Ramseyan partition relation. We also consider a selective version of paracompactness.
\end{abstract}

\section{Introduction}

Many mathematical notions are defined, or characterized, in terms of selection principles of the following two sorts: Let $S$ be an infinite set, and let $\mathcal{A}$ and $\mathcal{B}$ both be sets whose members are families of subsets of $S$.

Then $\mathrm{S}_{\mathrm{fin}}(\mathcal{A}, \mathcal{B})$ denotes the selection principle:

For each sequence $\left(A_{n}: n \in \mathbb{N}\right)$ of elements of $\mathcal{A}$ there is a sequence $\left(B_{n}: n \in \mathbb{N}\right)$ of finite sets such that for each $n \in \mathbb{N}$, $B_{n} \subset A_{n}$, and $\bigcup_{n \in \mathbb{N}} B_{n}$ is an element of $\mathcal{B}$.

Among topologists and set theorists the best known example of this sort of principle is known as the Menger property: For a topological space $X$, let $\mathcal{O}$ denote the collection of open covers of $X$. In [9], Hurewicz showed that a property for $X$ introduced by Menger in [13] is equivalent to the property $\mathrm{S}_{\text {fin }}(\mathcal{O}, \mathcal{O})$.

There is a natural game, denoted $\mathrm{G}_{\mathrm{fin}}(\mathcal{A}, \mathcal{B})$, associated with $\mathrm{S}_{\text {fin }}(\mathcal{A}$, $\mathcal{B})$. This game is played as follows: There is an inning per positive

Received March 4, 2004.

2000 Mathematics Subject Classification: 54D20, 54D45, 03E02, 91A44.

Key words and phrases: paracompact, locally compact, moving-off family, selection principles, Ramsey theory, game theory.

This work is supported by MNTR RS, Grant No 1233 and NSF grant DMS 9971282 . 
integer. In the $n$-th inning ONE chooses an $A_{n} \in \mathcal{A}$, and TWO responds with a finite set $B_{n} \subset A_{n}$. A play $A_{1}, B_{1} ; \cdots ; A_{n}, B_{n} ; \cdots$ is won by TWO if $\bigcup_{n \in \mathbb{N}} B_{n}$ is an element of $\mathcal{B}$; otherwise, ONE wins.

$\mathrm{S}_{1}(\mathcal{A}, \mathcal{B})$ denotes the selection principle:

For each sequence $\left(A_{n}: n \in \mathbb{N}\right)$ of elements of $\mathcal{A}$ there is a sequence $\left(b_{n}: n \in \mathbb{N}\right)$ such that for each $n \in \mathbb{N}, b_{n} \in A_{n}$ and $\left\{b_{n}: n \in \mathbb{N}\right\}$ is an element of $\mathcal{B}$.

The best known example of this sort of principle is known as the Rothberger property: For a topological space $X$, let $\mathcal{O}$ denote the collection of open covers of $X$. In [16], Rothberger introduced the property $\mathrm{S}_{1}(\mathcal{O}, \mathcal{O})$.

The corresponding game for this selection principle, denoted $\mathrm{G}_{1}(\mathcal{A}, \mathcal{B})$, is played similarly to the game $\mathrm{G}_{\text {fin }}(\mathcal{A}, \mathcal{B})$, but in the $n$-th inning TWO chooses an element $b_{n} \in A_{n}$ instead of a finite set $B_{n} \subset A_{n}$. A play $A_{1}, b_{1} ; \cdots ; A_{n}, b_{n} ; \cdots$ is won by TWO if and only if the set $\left\{b_{n}: n \in \mathbb{N}\right\}$ belongs to $\mathcal{B}$.

During the past few years it has been discovered, especially in the case when $\mathcal{A}$ and $\mathcal{B}$ are collections of certain kinds of open covers of a space, that these selection hypotheses can be equivalently stated in terms of Ramseyan partition relations (see $[10,11,17,18]$ ).

For positive integers $n$ and $k$ the symbol (called the ordinary partition symbol)

$$
\mathcal{A} \rightarrow(\mathcal{B})_{k}^{n}
$$

denotes the following statement:

For each $A \in \mathcal{A}$ and for each function $f:[A]^{n} \rightarrow\{1, \ldots, k\}$ there are a set $B \subset A, B \in \mathcal{B}$, and a $j \in\{1, \ldots, k\}$ such that for each $Y \in[B]^{n}, f(Y)=j$.

Here, as usual, the symbol $[A]^{n}$ denotes the set of $n$-element subsets of A.

Another partition symbol (called the Baumgartner-Taylor partition symbol)

$$
\mathcal{A} \rightarrow[\mathcal{B}\rceil_{k}^{2}
$$

denotes the statement:

For each $A$ in $\mathcal{A}$ and for each function $f:[A]^{2} \rightarrow\{1, \cdots, k\}$ there are a set $B \subset A, B \in \mathcal{B}$, a $j \in\{1, \cdots, k\}$ and a partition $B=\bigcup_{n \in \mathbb{N}} B_{n}$ of $B$ into pairwise disjoint finite sets such that for each $\{a, b\} \in[B]^{2}$ for which $a$ and $b$ are not from distinct $B_{n}$ 's, we have $f(\{a, b\})=j$. 
In this paper we consider yet another situation where an old concept (paracompactness) can be expressed in terms of notions that have become familiar in the theory of selection principles. This fact, together with the usual agenda in the study of selection principles, suggests a class of spaces which ought to have important topological properties.

\section{Gruenhage's characterization of paracompactness}

Our starting point is [6] where Gruenhage gives the following interesting characterization of paracompactness in locally compact $T_{2}$-spaces. Gruenhage defines for a space $X$ the following game, $\mathrm{G}^{*}(X)$ : Players ONE and TWO alternately choose compact subsets $K_{n}$ and $L_{n}$, respectively in inning $n$, where $n$ ranges over the positive integers. The rules of the game are that for each $n$ we have $K_{n} \cap L_{n}=\emptyset$. A play $\left(K_{1}, L_{1} ; \cdots ; K_{n}, L_{n}, \cdots\right)$ is a win for ONE if the set $\left\{L_{n}: n \in \mathbb{N}\right\}$ is locally finite; else, TWO wins.

TheOREM 1 (Gruenhage). For a locally compact $\mathrm{T}_{2}$ space $X$ the following are equivalent:

(1) $X$ is paracompact;

(2) ONE has a winning strategy in $\mathrm{G}^{*}(X)$.

\section{Converting to the arena of selection principles}

A family $\mathcal{M}$ of nonempty compact subsets of a space $X$ is a movingoff family if for each compact set $K$ there is a set $M \in \mathcal{M}$ such that $K \cap M=\emptyset([12])$.

If $X$ is a non-compact space, then its compact subsets form a movingoff family in $X$. Indeed, if $K \subset X$ is compact, choose any $x \in X \backslash K$ and one has $\{x\} \cap K=\emptyset$.

We define (for a space $X$ ):

- $\mathfrak{M}:=\{\mathcal{M}: \mathcal{M}$ is a moving-off family on $X\}$,

- $\mathfrak{L}:=\{\mathcal{L}: \mathcal{L}$ is an infinite locally finite family of compact subsets of $X\}$.

Having in mind Theorem 1, the next theorem gives another characterization of paracompactness in the class of locally compact Hausdorff spaces. 
THEOREM 2. For any non-compact topological space $X$ the following are equivalent:

(a) ONE has a winning strategy in $\mathrm{G}^{*}(X)$;

(b) TWO has a winning strategy in $\mathrm{G}_{\mathbb{1}}(\mathfrak{M}, \mathfrak{L})$.

Proof. $(a) \Rightarrow(b)$ : Let $\sigma$ be a winning strategy for ONE in $\mathrm{G}^{*}(X)$. We define a strategy $\tau$ for TWO in the game $G_{1}(\mathfrak{M}, \mathfrak{L})$. Let $\mathcal{M}_{1}$ be a movingoff family in $X$ chosen by ONE in the first move of the game $G_{1}(\mathfrak{M}, \mathfrak{L})$. TWO looks at $K_{1}=\sigma(\emptyset)$-the first move of ONE in $\mathrm{G}^{*}(X)$-and chooses $\tau\left(\mathcal{M}_{1}\right)=L_{1} \in \mathcal{M}_{1}$ such that $L_{1} \cap K_{1}=\emptyset$, which is possible because $\mathcal{M}_{1}$ is moving-off; this is a legitimate move for TWO in $\mathrm{G}^{*}(X)$. Let $\mathcal{M}_{2} \in \mathfrak{M}$ be the second move of ONE in $\mathrm{G}_{1}(\mathfrak{M}, \mathfrak{L})$. TWO looks at $K_{2}=\sigma\left(L_{1}\right)$ the second move of ONE in $\mathrm{G}^{*}(X)$-and chooses $\tau\left(\mathcal{M}_{1}, \mathcal{M}_{2}\right)=L_{2} \in \mathcal{M}_{2}$ such that $L_{2} \cap K_{2}=\emptyset$, and so on. Since $\sigma$ wins for ONE in $\mathrm{G}^{*}(X)$, $\left\{L_{1}, L_{2}, \ldots\right\} \in \mathfrak{L}$, i.e., $\tau$ wins for TWO in $\mathrm{G}_{1}(\mathfrak{M}, \mathfrak{L})$.

$(b) \Rightarrow(a)$ : Let $\tau$ be a winning strategy for TWO in $G_{1}(\mathfrak{M}, \mathfrak{L})$. We shall define a strategy $\sigma$ for ONE in $\mathrm{G}^{*}(X)$. Let

$\mathcal{E}_{1}:=\{K: K$ a compact subset of $X$ and for any $\mathcal{M} \in \mathfrak{M}, \tau(\mathcal{M}) \neq K\}$.

ClaIM: $\mathcal{E}_{1} \notin \mathfrak{M}$.

Suppose $\mathcal{E}_{1} \in \mathfrak{M}$. Then $\mathcal{E}_{1}$ is a legal move for ONE in $\mathrm{G}_{1}(\mathfrak{M}, \mathfrak{L})$. Consider TWO's response $C=\tau\left(\mathcal{E}_{1}\right)$. Then $C \in \mathcal{E}_{1}$ by rules of the game; on the other hand, by definition of $\mathcal{E}_{1}$ we have $C \notin \mathcal{E}_{1}$, and we have a contradiction.

Therefore, $\mathcal{E}_{1} \notin \mathfrak{M}$. Choose $K_{1}=\sigma(\emptyset)$ such that for each $K \in \mathcal{E}_{1}$, $K_{1} \cap K \neq \emptyset$. For any response $L_{1}$ by TWO (in $\left.\mathrm{G}^{*}(X)\right), L_{1} \cap K_{1}=\emptyset$ so that $L_{1} \notin \mathcal{E}_{1}$. Let $\mathcal{M}_{1}$ be a moving-off family such that $L_{1}=\tau\left(\mathcal{M}_{1}\right)$.

Define now

$$
\mathcal{E}_{2}:=\left\{K \in \mathcal{C}(X): \text { for any } \mathcal{M} \in \mathfrak{M}, \tau\left(\mathcal{M}_{1}, \mathcal{M}\right) \neq K\right\}
$$

where $\mathcal{C}(X)$ is the family of compact subsets of $X$. In a similar way as it was shown that $\mathcal{E}_{1}$ is not moving-off one may prove that $\mathcal{E}_{2}$ is not moving-off. Choose a compact set $K_{2} \subset X$ such that for all $K \in \mathcal{E}_{2}$, $K_{2} \cap K \neq \emptyset$; let $K_{2}=\sigma\left(L_{1}\right)$. If TWO's response is $L_{2}$, then $L_{2} \cap K_{2}=\emptyset$ implies $L_{2} \notin \mathcal{E}_{2}$. So, choose $\mathcal{M}_{2} \in \mathfrak{M}$ such that $L_{2}=\tau\left(\mathcal{M}_{1}, \mathcal{M}_{2}\right)$; and so on. TWO wins the game $G_{1}(\mathfrak{M}, \mathfrak{L})$, so that $\left\{\tau\left(\mathcal{M}_{1}\right), \tau\left(\mathcal{M}_{1}, \mathcal{M}_{2}\right), \ldots\right\} \equiv$ $\left\{L_{1}, L_{2}, \ldots\right\} \in \mathfrak{L}$, i.e., $\sigma$ is a winning strategy for ONE in $\mathrm{G}^{*}(X)$.

With minor modifications in the previous proof one proves: 
THEOREM 3. For any non-compact topological space $X$ the following are equivalent:

(a) TWO has a winning strategy in $\mathrm{G}^{*}(X)$;

(b) ONE has a winning strategy in $\mathrm{G}_{1}(\mathfrak{M}, \mathfrak{L})$.

EXAMPLE. Theorems 1 and 2 state that for locally compact $T_{2}$ spaces the following are equivalent:

1. The space is paracompact,

2. TWO has a winning strategy in the game $G_{1}(\mathfrak{M}, \mathfrak{L})$.

The assumption $T_{2}$ in this statement is important. Example 54 of [19] is locally compact non- $T_{2}$ and TWO has a winning strategy in $\mathrm{G}_{1}(\mathfrak{M}, \mathfrak{L})$, but the space is not paracompact.

\section{Basic properties of $\mathfrak{M}$ and $\mathfrak{L}$}

LEMMA 4. Each (infinite) element of $\mathfrak{L}$ is in $\mathfrak{M}$.

Proof. Let $\mathcal{L}$ be an element of $\mathfrak{L}$ which does not belong to $\mathfrak{M}$. There exists a compact set $K \subset X$ such that $K \cap L \neq \emptyset$ for each $L \in \mathcal{L}$. For each $x \in K$ choose a neighborhood $O_{x}$ of $x$ intersecting only finitely many members of $\mathcal{L}$. From the open cover $\left\{O_{x}: x \in K\right\}$ of $K$ choose a finite subcover $\left\{O_{x_{1}}, \ldots O_{x_{n}}\right\}$. It follows that $K$ intersects only finitely many elements from $\mathcal{L}$, which is a contradiction.

LEMMA 5. For each positive integer $k, \mathfrak{M} \rightarrow(\mathfrak{M})_{k}^{1}$.

Proof. Let $\mathcal{M}$ be a moving-off family in $X$ and $\mathcal{M}=\mathcal{M}_{1} \cup \cdots \cup$ $\mathcal{M}_{k}$. Assume no $\mathcal{M}_{i}, 1 \leq i \leq k$, is moving-off. Choose compact sets $K_{1}, \ldots, K_{k}$ in $X$ such that for each $i=1, \ldots, k$, for each $C \in \mathcal{M}_{i}$, $C \cap K_{i} \neq \emptyset$. Then the compact set $K=K_{1} \cup \cdots \cup K_{k}$ witnesses $\mathcal{M} \notin \mathfrak{M}$.

Lemma 6. For each $\mathcal{M}$ in $\mathfrak{M}$, and for each compact subset $K$ of $X$, $\mathcal{M}_{K}:=\{M \in \mathcal{M}: K \cap M=\emptyset\}$ is in $\mathfrak{M}$.

Proof. Suppose $\mathcal{M}_{K} \notin \mathfrak{M}$. Then there is a compact set $\Phi$ in $X$ such that $\Phi \cap M \neq \emptyset$ for each $M \in \mathcal{M}_{K}$. On the other hand, for the compact set $K \cup \Phi$ there is a set $M_{0} \in \mathcal{M}$ with $M_{0} \cap(K \cup \Phi)=\emptyset$. Thus $M_{0} \cap K=\emptyset$, which implies $M_{0} \in \mathcal{M}_{K}$, and $M_{0} \cap \Phi=\emptyset$ and we have a contradiction.

Lemma 7. If $f: X \rightarrow Y$ is a perfect mapping and $X$ satisfies $\mathrm{S}_{1}(\mathfrak{M}, \mathfrak{L})$, then $Y$ also has the same property. 
Proof. Let $\left(\mathcal{M}_{n}: n \in \mathbb{N}\right)$ be a sequence of moving-off families in $Y$. Using perfectness of $f$ it is easy to check that $\left(f^{\leftarrow}\left(\mathcal{M}_{n}\right): n \in \mathbb{N}\right)$ is a sequence of moving-off families in $X$. Since $X$ has property $\mathrm{S}_{1}(\mathfrak{M}, \mathfrak{L})$, for each $n$ there is a set $L_{n} \in \mathcal{M}_{n}$ such that $\left\{f^{\leftarrow}\left(L_{n}\right): n \in \mathbb{N}\right\}$ is a locally finite family (of compact sets) in $X$. Perfect mappings preserve local finiteness (Lemma 3.10.11 in [4]) so that $\left\{L_{n}: n<\infty\right\}$ is a locally finite family in $Y$.

\section{Consequences of $S_{1}(\mathfrak{M}, \mathfrak{L})$}

Lemma 8 . Let $X$ be locally compact $T_{2}$ but not compact. If $X$ satisfies $S_{1}(\mathfrak{M}, \mathfrak{L})$, then each element of $\mathfrak{M}$ has a countably infinite subset which is an element of $\mathcal{L}$.

Proof. Let $\mathcal{M}$ be an element of $\mathfrak{M}$. We must find a countably infinite subset $\mathcal{N}$ of $\mathcal{M}$ such that $\mathcal{N}$ is in $\mathfrak{L}$. We show something slightly more general first:

For each sequence $\left(\mathcal{K}_{n}: n \in \mathbb{N}\right)$ of elements of $\mathfrak{M}$ there is a sequence $\left(K_{n}: n \in \mathbb{N}\right)$ such that for each $n$ we have $K_{n} \in \mathcal{K}_{n}$, $\left\{K_{n}: n \in \mathbb{N}\right\}$ is in $\mathfrak{L}$, and is infinite.

Let $\left(\mathcal{K}_{n}: n \in \mathbb{N}\right)$ be a sequence of elements of $\mathfrak{M}$. Applying $S_{1}(\mathfrak{M}, \mathfrak{L})$ we choose for each $n$ an $L_{n} \in \mathcal{K}_{n}$ such that $\left\{L_{n}: n \in \mathbb{N}\right\}$ is in $\mathfrak{L}$. If this set of $L_{n}$ 's is infinite we are done. So assume it is finite, and choose $n_{1}$ so that $\left\{L_{n}: n \in \mathbb{N}\right\}=\left\{L_{j}: j \leq n_{1}\right\}$. Now $K_{1}=\bigcup_{j \leq n_{1}} L_{j}$ is a compact set.

Choose an open set $V_{1}$ such that $K_{1} \subset V_{1}$, and $\overline{V_{1}}$ is compact; this is possible because of local compactness of $X$. Then for each $j>n_{1}$ put $\mathcal{K}_{j}^{2}=\left\{K \in \mathcal{K}_{j}: K \cap \overline{V_{1}}=\emptyset\right\}$.

By Lemma 6 , the sequence $\left(\mathcal{K}_{j}^{2}: n_{1}<j<\infty\right)$ is a sequence of members of $\mathfrak{M}$. Again, apply $\mathrm{S}_{1}(\mathfrak{M}, \mathfrak{L})$ to this sequence to obtain a sequence $\left(L_{j}^{2}: n_{1}<j<\infty\right)$ where for each such $j$ we have $L_{j}^{2} \in \mathcal{K}_{j}^{2}$, and $\left\{L_{j}^{2}: n_{1}<j<\infty\right\}$ is in $\mathfrak{L}$. If this set is infinite, we are done. Else, there is an $n_{2}>n_{1}$ such that $\left\{L_{j}^{2}: n_{1}<j<\infty\right\}=\left\{L_{j}^{2}: n_{1}<j \leq n_{2}\right\}$. Then define $K_{2}=\bigcup_{n_{1}<j \leq n_{2}} L_{j}^{2}$.

Since $K_{2}$ is compact and disjoint from the compact set $\overline{V_{1}}$, again by local compactness of $X$, we find an open set $V_{2} \supset K_{2}$ such that $\overline{V_{2}}$ is compact and also disjoint from $\overline{V_{1}}$. For each $j>n_{2}$ define: $\mathcal{K}_{j}^{3}=\{K \in$ $\left.\mathcal{K}_{j}^{2}: K \cap \overline{V_{2}}=\emptyset\right\}$, and so on. 
Either at some finite stage of this construction an infinite selector for the original sequence of $\mathcal{K}_{n}$ 's is obtained, or else the collection $\left\{L_{j}: j \leq\right.$ $\left.n_{1}\right\} \cup\left\{L_{j}^{2}: n_{1}<j \leq n_{2}\right\} \cup \cdots$ is an infinite, locally finite (as witnessed by the $V_{n}$ 's) selector.

TheOREM 9. For $X$ non-compact, but locally compact $T_{2}$, the following are equivalent:

(1) ONE has no winning strategy in the game $\mathrm{G}_{1}(\mathfrak{M}, \mathfrak{L})$;

(2) $\mathrm{S}_{1}(\mathfrak{M}, \mathfrak{L})$;

(3) $\mathrm{S}_{\mathrm{fin}}(\mathfrak{M}, \mathfrak{L})$;

(4) ONE has no winning strategy in the game $\mathrm{G}_{\mathrm{fin}}(\mathfrak{M}, \mathfrak{L})$.

Proof. (1) $\Rightarrow(2)$ : By standard arguments.

$(2) \Rightarrow(1)$ : Let $X$ be as in the hypotheses and let $\sigma$ be a strategy for ONE in the game $\mathrm{G}_{1}(\mathfrak{M}, \mathfrak{L})$. We show that there is a $\sigma$-play which is lost by ONE. To this end we construct compact sets $\left(L_{n_{1}, \cdots, n_{k}}: n_{1}, \cdots, n_{k}<\right.$ $\infty)$ such that:

(i) $\left(L_{n}: n \in \mathbb{N}\right)$ is infinite and in $\mathfrak{L}$;

(ii) For each $n_{1}, \cdots, n_{k},\left(L_{n_{1}, \cdots, n_{k}, n}: n \in \mathbb{N}\right)$ is infinite and in $\mathfrak{L}$.

Namely: $\left(L_{n}: n \in \mathbb{N}\right)$ is a subset of $\sigma(\emptyset)$, the first move of ONE. An infinite set like this exists by the preceding lemma. Next, for each $n$, pretend $L_{n}$ is TWO's move and consider ONE's response $\sigma\left(L_{n}\right)$, an element of $\mathfrak{M}$. Applying the preceding lemma again we find an infinite set $\left(L_{n, k}: k<\infty\right)$ in $\mathfrak{L}$ which is a subset of $\sigma\left(L_{n}\right)$. Then with $L_{n_{1}}$ and $L_{n_{1}, n_{2}}$ given, consider $\sigma\left(L_{n_{1}}, L_{n_{1}, n_{2}}\right)$, a move by ONE and so an element of $\mathfrak{M}$. Applying Lemma 8 once more we find an infinite set $\left(L_{n_{1}, n_{2}, k}: k<\infty\right)$ in $\mathfrak{L}$, contained in $\sigma\left(L_{n_{1}}, L_{n_{1}, n_{2}}\right)$, and so on.

Now we construct a play for TWO against $\sigma$, which defeats $\sigma$. To begin, TWO chooses $L_{1}$. By local compactness of $X$ choose an open set $V_{1} \supset L_{1}$ with $\overline{V_{1}}$ compact. Applying Lemma 4, choose an $n_{2}$ so that $L_{1, n_{2}} \cap \overline{V_{1}}=\emptyset$. Then choose an open set $V_{2} \supset L_{1, n_{2}}$ with $\overline{V_{2}}$ compact and disjoint from $\overline{V_{1}}$. Again use Lemma 4 and choose $n_{3}$ so that $L_{1, n_{2}, n_{3}}$ is disjoint from $\overline{V_{1}} \cup \overline{V_{2}}$, and so on.

The so constructed sequence

$$
L_{1}, L_{1, n_{2}}, L_{1, n_{2}, n_{3}}, \cdots
$$

is a legal sequence of moves by TWO and is in $\mathfrak{L}$ - whence ONE lost the corresponding play, even though ONE was using the strategy $\sigma$.

$(3) \Rightarrow(2)$ : Let $\left(\mathcal{M}_{n}: n<\infty\right)$ be a sequence of elements of $\mathfrak{M}$. We define for each $n$ : 


$$
\mathcal{N}_{n}:=\left\{M_{1} \cup \cdots \cup M_{n}: 1 \leq i \leq n, M_{i} \in \mathcal{M}_{i}\right\} .
$$

Now for each $n$ choose a finite set $\mathcal{F}_{n} \subset \mathcal{N}_{n}$ such that $\bigcup_{n \in \mathbb{N}} \mathcal{F}_{n}$ is an element of $\mathfrak{L}$. Let $\left\{k_{1}, \cdots, k_{n}, \cdots\right\}$ enumerate the infinite set $\left\{k: \mathcal{F}_{k} \neq\right.$ $\emptyset\}$. Write

Now put

$$
F_{k_{j}}=M_{1}^{\dot{j}} \cup \cdots \cup M_{k_{j}}^{j} \text { for all } j \text {. }
$$

$$
L_{j}= \begin{cases}M_{j}^{1} & \text { if } j \leq k_{1}, \\ M_{j}^{i} & \text { if } k_{i-1}<j \leq k_{i} .\end{cases}
$$

Each $L_{j}$ is an element of $\mathcal{M}_{j}$ and $\left\{L_{j}: j<\infty\right\} \in \mathfrak{L}$. So, we found a sequence witnessing $\mathrm{S}_{1}(\mathfrak{M}, \mathfrak{L})$.

$(2) \Rightarrow(3)$ is obvious and $(4) \Rightarrow(3)$ is standard.

$(3) \Rightarrow(4)$ : It is proven similarly to the proof of $(2) \Rightarrow(1)$.

CoROLlaRY 10. If $X$ is a locally compact, but not compact, $T_{2}$-space such that $\mathrm{S}_{1}(\mathfrak{M}, \mathfrak{L})$ holds, then for each positive integer $k, \mathfrak{M} \rightarrow(\mathfrak{L})_{k}^{2}$.

Proof. It follows from Lemma 5 and Theorem 1 from [11].

Using this result, by standard induction on $n$ and $k$, one obtains also

COROLLARY 11. If $X$ is a locally compact, but not compact, $T_{2}$-space such that $\mathrm{S}_{1}(\mathfrak{M}, \mathfrak{L})$ holds, then for each $n$ and $k$ in $\mathbb{N}, \mathfrak{M} \rightarrow(\mathfrak{L})_{k}^{n}$.

\section{Selection hypothesis from Ramseyan hypothesis-a differ- ent version of "selectable pair"}

In [11], we introduced the notion of selectable pair $(\mathcal{A}, \mathcal{B})$ in order to find general results showing when partition relations imply selection hypotheses. We define now another version of that notion.

A pair $(\mathcal{A}, \mathcal{B})$ is "dually selectable" if:

$(D S 1) \mathcal{A}$ and $\mathcal{B}$ are subsets of $\mathcal{P}(\mathcal{P}(S))$;

$(D S 2)$ If $\mathcal{U} \in \mathcal{A}$ is countable and $f: \mathcal{U} \rightarrow \mathbb{N}$ is a function, and $\left(\mathcal{V}_{n}: n \in \mathbb{N}\right)$ is a sequence of elements of $\mathcal{A}$, then also $\{A \cup B: A \in \mathcal{U}, B \in$ $\left.\mathcal{V}_{f(A)}\right\}$ is an element of $\mathcal{A}$.

(DS3) Each element of $\mathcal{A}$ has a countable subset which is an element of $\mathcal{A}$.

(DS4) For each $A \in \mathcal{A}$ and for each $K \in A, \mathcal{P}(\{Z \subset S: K \subset Z\}) \cap \mathcal{B}=\emptyset$.

$(D S 5)$ If $B$ is in $\mathcal{B}$ and $F \subset B$ is finite, then $B \backslash F$ is in $\mathcal{B}$.

(DS6) If $C$ is a countable set of subsets of $S$ such that 
(1) $(\exists B \in \mathcal{B})(\{b \in B:(\exists X \in C)(X \subset b)\} \in \mathcal{B})$ and

(2) $(\forall X \in C)(\exists A \in \mathcal{A})(X \in A)$

then $C$ is a member of $\mathcal{B}$.

For locally compact, but non-compact $T_{2}$-spaces, $(\mathfrak{M}, \mathfrak{L}$ ) (defined in the beginning of the paper) is a "dually selectable pair".

Following proofs of Theorems 4 and 5 in [11], and making the necessary small adjustments, one obtains:

TheOREM 12. If $(\mathcal{A}, \mathcal{B})$ is a "dually selectable pair", then:

(1) If for each $k \in \mathbb{N}, \mathcal{A} \rightarrow\lceil\mathcal{B}\rceil_{k}^{2}$, then $\mathrm{S}_{\text {fin }}(\mathcal{A}, \mathcal{B})$ holds.

(2) If for each $k \in \mathbb{N}, \mathcal{A} \rightarrow(\mathcal{B})_{k}^{2}$, then $\mathrm{S}_{1}(\mathcal{A}, \mathcal{B})$ holds.

The last theorem, Theorem 9, Lemma 5 and Theorem 1 from [11] give the following important corollary.

Corollary 13. Let $X$ be a non-compact, locally compact $T_{2}$-space. Then the following are equivalent:

(1) $X$ satisfies $\mathrm{S}_{1}(\mathfrak{M}, \mathfrak{L})$;

(2) ONE has no winning strategy in the game $\mathrm{G}_{1}(\mathfrak{M}, \mathfrak{L})$;

(3) For each $n$ and $k$ in $\mathbb{N}, \mathfrak{M} \rightarrow(\mathfrak{L})_{k}^{n}$.

Let us mention that this result can be compared with a similar result from [7] (Theorem 2.3). The game in that theorem is similar to our game $\mathrm{G}_{1}(\mathfrak{M}, \mathfrak{L})$, and the part (i) from [7] is actually our (3) with $n=k=1$.

\section{Another selection principle and paracompactness}

In 1973, W. Haver[8] introduced a covering property for metric spaces called property C. In [1], Addis and Gresham reformulated the original definition of $\mathrm{C}$ in a form much more convenient for use in general topology:

A topological space $X$ has property $C$ if for each sequence $\left(\mathcal{U}_{n}: n \in \mathbb{N}\right)$ of open covers of $X$ there is a sequence $\left(\mathcal{V}_{n}: n \in \mathbb{N}\right)$ of families of open subsets of $X$ satisfying the following three conditions:

(1) For each $n \in \mathbb{N}, \mathcal{V}_{n}$ is a disjoint family;

(2) For each $n \in \mathbb{N}, \mathcal{V}_{n}<\mathcal{U}_{n}$;

(3) $\bigcup_{n \in \mathbb{N}} \mathcal{V}_{n}$ is an open cover of $X$.

In [2], this property was denoted by $S_{c}(\mathcal{O}, \mathcal{O})$. [2] also defined and studied as a general selection principle $\mathrm{S}_{c}(\mathcal{A}, \mathcal{B})$, where $\mathcal{A}$ and $\mathcal{B}$ are collections of families of subsets of a space $X$. 
We define now the following general selection principle.

Let $X$ be a topological space and let $\mathcal{A}$ and $\mathcal{B}$ denote collections whose elements are families of open subsets of $X$. The $\operatorname{symbol} \mathrm{S}_{\mathrm{If}}(\mathcal{A}, \mathcal{B})$ denotes the following statement:

For each sequence $\left(\mathcal{U}_{n}: n \in \mathbb{N}\right)$ of elements of $\mathcal{A}$ there is a sequence $\left(\mathcal{V}_{n}: n \in \mathbb{N}\right)$ such that for each $n \in \mathbb{N}, \mathcal{V}_{n}$ is a locally finite family with $\mathcal{V}_{n}<\mathcal{U}_{n}$ (= for each $V \in \mathcal{V}_{n}$ there is a $U \in \mathcal{U}_{n}$ with $\left.V \subset U\right)$ and $\bigcup_{n \in \mathbb{N}} \mathcal{V}_{n} \in \mathcal{B}$.

It is clear that $\mathrm{S}_{\text {fin }}(\mathcal{A}, \mathcal{B})$ implies $\mathrm{S}_{\mathrm{If}}(\mathcal{A}, \mathcal{B})$.

Let us introduce the following notation (for a space $X$ ):

- $\mathcal{O}$ the collection of open covers of $X$;

- $\Omega$ the collection of $\omega$-covers of $X$; an open cover $\mathcal{U}$ of $X$ is an $\omega$-cover if $X$ is not a member of $\mathcal{U}$ and for each finite subset $F$ of $X$ there is a $U \in \mathcal{U}$ such that $F \subset U$.

- $\Gamma$ the collection of $\gamma$-covers of $X$; an open cover $\mathcal{U}$ of $X$ is called a $\gamma$-cover if it is infinite and for each $x \in X$ the set $\{U \in \mathcal{U}: x \notin U\}$ is finite.

From the definitions it follows that every paracompact space (no separation axiom is assumed) satisfies $\mathrm{S}_{\mathrm{If}}(\mathcal{O}, \mathcal{O})$.

We shall see now that in the class of regular spaces paracompactness can be characterized by $\mathrm{S}_{\mathrm{lf}}(\mathcal{A}, \mathcal{B})$ properties.

Theorem 14. Let $X$ be a regular space. The following are equivalent:

(a) $X$ is paracompact;

(b) $X$ satisfies $\mathrm{S}_{\mathrm{If}}(\mathcal{O}, \mathcal{O})$;

(c) $X$ satisfies $\mathrm{S}_{\mathrm{lf}}(\Omega, \mathcal{O})$.

Proof. $(a) \Rightarrow(b)$ : Trivial.

$(b) \Rightarrow(c)$ : It follows from the fact $\Omega \subset \mathcal{O}$.

$(c) \Rightarrow(b)$ : Let $\left(\mathcal{U}_{n}: n \in \mathbb{N}\right)$ be a sequence of open covers of $X$. Let $\mathbb{N}=N_{1} \cup N_{2} \cup \ldots$ be a partition of $\mathbb{N}$ into countably many pairwise disjoint infinite subsets. For every $n \in \mathbb{N}$ let $\mathcal{V}_{n}$ be the set of elements of the form $U_{n_{1}} \cup \cdots \cup U_{n_{k}}, k \in \mathbb{N}, n_{1}<n_{2}<\cdots<n_{k} \in N_{n}, U_{n_{i}} \in \mathcal{U}_{n_{i}}$ for all $i=1,2, \ldots, k$. The sequence $\left(\mathcal{V}_{n}: n \in \mathbb{N}\right)$ is a sequence of $\omega$-covers of $X$. Apply $\mathrm{S}_{\text {lf }}(\Omega, \mathcal{O})$ to the later sequence to find a sequence $\left(\mathcal{W}_{n}: n \in \mathbb{N}\right)$ such that for each $n \mathcal{W}_{n}$ is locally finite, $\mathcal{W}_{n}<\mathcal{V}_{n}$ and $\bigcup_{n \in \mathbb{N}} \mathcal{W}_{n}$ is an open cover of $X$. For each $n$ and each $W \in \mathcal{W}_{n}$ choose $V_{W} \in \mathcal{V}_{n}$ such that $W \subset V_{W}$. If $V_{W}=U_{n_{1}} \cup \cdots \cup U_{n_{k}}, n_{1}<n_{2}<\cdots<n_{k} \in N_{n}$, $U_{n_{i}} \in \mathcal{U}_{n_{i}}$ for $i=1,2, \ldots, k$, then choose all the sets $U_{n_{s}} \cap W$ which 
are nonempty and denote by $H(W)$ the family of sets chosen in this way. Let $\mathcal{H}_{n}=\cup\left\{H(W): W \in \mathcal{W}_{n}\right\}$. Then the sequence $\left(\mathcal{H}_{n}: n \in \mathbb{N}\right)$ witnesses for $\left(\mathcal{U}_{n}: n \in \mathbb{N}\right)$ that $X$ satisfies $\mathrm{S}_{\mathrm{lf}}(\mathcal{O}, \mathcal{O})$.

(b) $\Rightarrow(a)$ : Let $\mathcal{U}$ be an open cover of $X$. Applying (b) to the sequence $\left(\mathcal{U}_{n}=\mathcal{U}: n \in \mathbb{N}\right)$ we find a $\sigma$-locally finite refinement of $\mathcal{U}$. By Michael's characterization of paracompactness ([4]) we conclude that $X$ is paracompact.

The following two theorems show that for some "nice" families of open covers the selection principle $S_{\text {lf }}$ coincides with the principle $S_{\text {fin }}$.

ThEOREM 15. $\mathrm{S}_{\mathrm{If}}(\Omega, \Gamma)$ implies $\mathrm{S}_{\mathrm{fin}}(\Omega, \Gamma)=\mathrm{S}_{1}(\Omega, \Gamma)$.

Proof. Let $\left(\mathcal{U}_{n}: n \in \mathbb{N}\right)$ be a sequence of $\omega$-covers of $X$. Choose for each $n$ a locally finite collection $\mathcal{V}_{n}<\mathcal{U}_{n}$ such that $\bigcup_{n \in \mathbb{N}} \mathcal{V}_{n} \in \Gamma$. We show that each $\mathcal{V}_{n}$ is in fact finite. Suppose there is some $k$ for which $\mathcal{V}_{k}$ is infinite. Take any $x \in X$. Then, $x$ belongs to all but finitely many elements of $\bigcup_{n \in \mathbb{N}} \mathcal{V}_{n}$, hence to infinitely many members of $\mathcal{V}_{k}$. On the other hand, there is a neighborhood $O_{x}$ of $x$ intersecting finitely many elements of $\mathcal{V}_{k}$. A contradiction. $\mathrm{S}_{\mathrm{fin}}(\Omega, \Gamma)=\mathrm{S}_{1}(\Omega, \Gamma)$ was proved in [10].

THEOREM 16. $\mathrm{S}_{\mathrm{If}}(\Omega, \Omega)$ implies $\mathrm{S}_{\text {fin }}(\Omega, \Omega)$.

Proof. Let $\left(\mathcal{U}_{n}: n \in \mathbb{N}\right)$ be a sequence of $\omega$-covers of $X$. For each $n$ pick a locally finite collection $\mathcal{V}_{n}<\mathcal{U}_{n}$ such that $\bigcup_{n \in \mathbb{N}} \mathcal{V}_{n}$ is an $\omega$-cover of $X$. We prove that each $\mathcal{V}_{n}$ is finite. Let $x$ be any point in $X$. Then for each $n, x$ belongs to finitely many members of $\mathcal{V}_{n}$. Let $K$ be a finite subset of $X$. The set $K \cup\{x\}$ is finite, hence there are a natural number $m$ and $V \in \mathcal{V}_{m}$ with $K \cup\{x\} \subset V$. This means that $K$ must be actually contained in one of finitely many members in $\mathcal{V}_{m}$ that contain $x$.

\section{References}

[1] D. F. Addis and J. H. Gresham, A class of infinite-dimensional spaces, Part I: Dimension theory and Alexandroff's problem, Fund. Math. 101 (1978), 195-205.

[2] L. Babinkostova, Selection principles in topology, Doctoral dissertation, Skopje, 2001, (in Macedonian).

[3] J. E. Baumgartner and A. D. Taylor, Partition theorems and ultrafilters, Trans. Amer. Math. Soc. 241 (1978), 283-309.

[4] R. Engelking, General Topology, PWN, Warszawa, 1977.

[5] F. Galvin, Indeterminacy of point-open games, Bull. Acad. Polon. Sci. Sér. Sci. Math. Astronom. Phys. 26 (1978), 445-448.

[6] G. Gruenhage, Games, covering properties and Eberlein compacts, Topology Appl. 23 (1986), 291-297. 
[7] G. Gruenhage and D. K. Ma, Baireness of $C_{k}(X)$ for locally compact $X$, Topology Appl. 80 (1997), 131-139.

[8] W. Haver, A covering property for metric spaces, Proc. Topology Conference at Virginia Polytechnic Institute and State University, March 22-29, 1973, R. F. Dickman, Jr. and P. Fletcher (eds.), Lectures Notes in Math. 375 (1974).

[9] W. Hurewicz, Über eine Verallgemeinerung des Borelschen Theorems, Math. Z. 24 (1925), 401-421.

[10] W. Just, A. W. Miller, M. Scheepers, and P. J. Szeptycki, The combinatorics of open covers II, Topology Appl. 73 (1996), 241-266.

[11] Lj. D. R. Kočinac and M. Scheepers, The combinatorics of open covers (VII): Groupability, Fund. Math. 179 (2003), 131-155.

[12] D. K. Ma, The Cantor tree, the $\gamma$-property, and Baire function spaces, Proc. Amer. Math. Soc. 119 (1993), 903-913.

[13] K. Menger, Einige Überdeckungssätze der Punktmengenlehre, Sitzungsberichte Abt. 2a, Mathematik, Astronomie, Physik, Meteorologie und Mechanik (Wiener Akademie, Wien) 133 (1924), 421-444.

[14] A. W. Miller and D. H. Fremlin, On some properties of Hurewicz, Menger and Rothberger, Fund. Math. 129 (1988), 17-33.

[15] F. P. Ramsey, On a problem of formal logic, Proc. London Math. Soc. 30 (1930), 264-286.

[16] F. Rothberger, Eine Vershärfung der Eigenschaft C, Fund. Math. 30 (1938), $50-55$.

[17] M. Scheepers, Combinatorics of open covers I: Ramsey theory, Topology Appl. 69 (1996), 31-62.

[18] $\_$Open covers and partition relations, Proc. Amer. Math. Soc. 127 (1999), $577-581$.

[19] L. A. Steen and J. A. Seebach, Jr., Counterexamples in Topology, SpringerVerlag, New York Inc. $1978_{2}$.

Liljana Babinkostova

Faculty of Natural Sciences and Mathematics

Institute of Mathematics

P.O. Box 162 91000, Skopje

E-mail: babinkostova@yahoo.com

Ljubiša D.R. Kočinac

Faculty of Sciences

University of Niš

Višegradska 33, 18000 Niš, Serbia

E-mail: lkocinac@ptt.yu 
Marion Scheepers

Department of Mathematics

Boise State University

Boise, Idaho 83725 , U.S.A.

E-mail: marion@diamond.boisestate.edu 